\title{
Deep survey for designing a vaccine against SARS-CoV-2 and its new mutations
}

\author{
Bahareh Vakili $^{1,2} \cdot$ Ashkan Bagheri $^{1} \cdot$ Manica Negahdaripour ${ }^{1,3}$ (D) \\ Received: 1 January 2021 / Accepted: 16 July 2021 / Published online: 17 August 2021 \\ (C) Institute of Molecular Biology, Slovak Academy of Sciences 2021
}

\begin{abstract}
The ongoing global pandemic caused by severe acute respiratory syndrome coronavirus-2 (SARS-CoV-2) has prompted worldwide vaccine development. Several vaccines have been authorized by WHO, FDA, or MOH of different countries. However, issues such as need for cold chain, price, and most importantly access problems have limited vaccine usage in some nations especially developing countries. Moreover, the vast global demand justifies further attempts for vaccine development. Multiepitope polypeptide vaccines enjoy several key features including safety and lower production and transfer costs and could be designed by in silico tools. Spike protein $(\mathrm{S})$, membrane protein $(\mathrm{M})$, and nucleocapsid protein $(\mathrm{N})$, the three major structural proteins of SARS-CoV-2, are ideal candidates for epitope selection. ORF3a (open reading frame3a), a transmembrane protein with pro-apoptotic functions, could be another proper target. Thus, a novel multi-epitope vaccine against SARS-CoV-2 was designed using these four proteins and LL37, a TLR3 agonist adjuvant, through different immunoinformatics and bioinformatics tools. The proposed multi-epitope vaccine is expected to induce robust humoral and cellular immune responses against SARSCoV-2 with a population coverage of $76.92 \%$ due to containing different immunodominant epitopes and LL37 adjuvant. Selecting epitopes derived from one functional and three structural proteins suggests the protective ability of the vaccine irrespective of probable virus mutations. The computationally observed proper interaction of LL37 with TLR3 implies its ability to induce immune responses effectively. Besides, it showed acceptable structural and physicochemical properties. The in-silico cloning results predicted its high efficiency production in Escherichia coli. Future experimental studies could further confirm its immunological efficacy.
\end{abstract}

Keywords Adjuvant $\cdot$ Coronavirus $\cdot$ In silico $\cdot$ Multi-epitope $\cdot$ Immunoinformatics, COVID-19

\section{Introduction}

Severe acute respiratory syndrome coronavirus-2 (SARS-CoV-2) has grown to an ongoing global pandemic, following the first reported case at the end of 2019 in Wuhan, Hubei province, China, and has caused serious healthcare and disease control

Bahareh Vakili and Ashkan Bagheri contributed equally to this work.

Manica Negahdaripour

negahdaripour@sums.ac.ir

1 Pharmaceutical Sciences Research Center, Shiraz University of Medical Sciences, Shiraz, Iran

2 Allergy Research Center, Shiraz University of Medical Sciences, Shiraz, Iran

3 Department of Pharmaceutical Biotechnology, School of Pharmacy, Shiraz University of Medical Sciences, P.O. Box 71345-1583, Shiraz, Iran concerns worldwide (Mosaddeghi et al. 2020). As of 21 May 2021, over 165 million cases of coronavirus disease 2019 (COVID-19) have been confirmed leading to over 3.4 million deaths (WHO, 21 May 2021). SARS-CoV-2 is a $\beta$-coronavirus from the family Coronaviridae (Negahdaripour 2020). The genome sequence of SAR-CoV-2 is nearly $80 \%$ similar to that of SARS-CoV, another $\beta$-coronavirus responsible for SARS epidemic in 2003 (Zhou et al. 2020).

Many studies have been carried out to identify and investigate the structural and functional proteins of SARS-CoV-2, which could lead to a better understanding of the virus pathology, virulence, and even emergence of new therapeutic and preventive options. SARS-CoV-2 transcriptome encodes four main structural proteins: spike protein (S), membrane protein $(\mathrm{M})$, envelope protein $(\mathrm{E})$, and nucleocapsid protein $(\mathrm{N})$ (Drosten et al. 2003), which are commonly present in all coronaviruses alongside an open reading frame (ORF1a/ ORF1ab) (Michel et al. 2020). While M and E proteins of 
the virus participate in particle assembly and release of the virus (Masters 2006), the S1 subunit of S protein, binds to host cell receptors, and the S2 subunit participates in the process of fusion between viral and host cell membranes thereby facilitating virus entry to host cells (Owji et al. 2020). The key receptor for the receptor binding domain (RBD), which is present in the $\mathrm{S}$ protein, is called angiotensin converting enzyme-2 (ACE2). ACE2 is widely distributed in various human organs such as kidneys, liver, small intestine, and lungs (Hamming et al. 2004). Previous publications have discussed the functions of SARS-CoV-2 accessory proteins, including ORF3a, 6, 7a, 7b (Gordon et al. 2020), its pathophysiology, as well as the signaling and immune response pathways involved in this viral infection (Bagheri et al. 2020).

The recent pandemic outbreak prompted worldwide vaccine development, as urgent tactics are needed to prevent further transmission of the virus. Many research groups around the world started development of vaccine candidates, some of which are already approved and some others are in pre-clinical and clinical stages of development (Kaur and Gupta 2020). The US Food and Drug Administration (FDA) issued emergency use authorization (EUA) for Pfizer-BioNTech, Moderna, and Janssen COVID-19 vaccines (FDA US 2020a, b, 2021), while more than 10 vaccines have received an NRA (national regulatory authority) approval to be used in at least one country, and vaccination with these vaccines has been started in some countries (WHO website, accessed on 22 May 2021). However, some challenges such as cold chain maintenance, access issues, and high costs have dampened vaccine usage particularly in developing countries. Moreover, the vast global demand justifies further attempts for development of more vaccines (Negahdaripour, 2021). The efficacy and safety issues are the main challenges for vaccine developers as ever.

Multi-epitope polypeptide vaccines are shown to be safe, needless of complex storage conditions, with lower production and transfer costs (Negahdaripour et al. 2017a, 2018). Still, low immunogenicity is one of the main burdens in the development of polypeptide vaccines. To tackle this issue, employment of adjuvants, as immune response enhancers, could provide beneficiary outcomes by triggering a stronger immune response with fewer doses of the antigen (Coffman et al. 2010).

During recent years, administration of various bioinformatics tools has proved to be a cost-effective and time-saving strategy in modern structural vaccinology, which supports better antigen selection and design (Negahdaripour et al. 2018). In this regard, in silico approaches help to predict the immunogenic epitope regions of various antigenic proteins in order to generate an effective immunological response. Furthermore, the expression level of a gene can be predicted and optimized through in silico cloning before growing the microorganism in vitro (Negahdaripour et al. 2017a, Samad et al. 2020, Yang et al. 2021).
In this study, various bioinformatics servers and computational tools would be employed to design a novel multiepitope against SARS-CoV-2 using epitope regions from the $\mathrm{S}, \mathrm{M}$, and $\mathrm{N}$ proteins as well as the ORF3a of SARS-CoV-2. A toll-like receptor (TLR) 3 agonist would also be administered as the vaccine adjuvant to further increase the immunogenic potential of the construct.

\section{Materials and methods}

\section{Sequence retrieval}

The complete amino acid sequences of $\mathrm{S}$ protein (Accession no. YP_009724390.1), M protein (Accession no. YP_009724393.1), N protein (Accession no. YP_009724397.2), and ORF3a (Accession no. YP_009724391.1) were retrieved from the National Centre for Biotechnology Information (NCBI) at www.ncbi.nlm. nih.gov in FASTA format. L L 37 (LLGDFFRKSKEKIGKEFKRIVQRIKDFLRNLVPRTES) was applied as a TLR3 agonist (Lai et al. 2011b). Moreover, the complete amino acid sequence and structure of TLR3 was retrieved from the PDB database at https://www.rcsb.org/pdb/ home/home.do (PDB code: 1ZIW).

\section{Identification of immunodominant epitopes}

\section{HLA class I and II binding epitope prediction}

For human leukocyte antigen (HLA) class I binding epitope prediction, we selected the 16 most frequent HLA class I alleles in the worldwide population, including HLA$A * 01: 01$, HLA-A*02:01, HLA-A*03:01, HLA-A*11:01, HLA-B*14:02, HLA-A*23:01, HLA-A*24:02, HLA$A * 31: 01$, HLA-A*68:01, HLA-B*07:02, HLA-B*08:01, HLA-B*27:02, HLA-B*27:05, HLA-B*35:01, HLA$B * 40: 01$, HLA-B*44:03. The high-score 9-mer epitopes were identified using immune epitope databases (IEDB) (http:// tools.iedb.org/mhci). IEDB is an online server that applies different methods for the prediction of HLA class I and II binding epitopes. (Kim et al. 2012). Furthermore, using the IEDB server, the high-ranked 15-mer HLA class II binding epitopes for 22 most frequent HLA class II alleles in the worldwide population were predicted, including $\mathrm{H}$ DRB1*01:01, DRB1*01:02, DRB1*03:01, DRB1*04:01, DRB1*04:05, DRB1*07:01, DRB1*07:03, DRB1*08:02, DRB1*08:17, DRB1*09:01, DRB1*12:01, DRB1*11:01, DRB1*11:06, DRB1*13:02, DRB1*13:03, DRB1*13:05, DRB1*14:01, DRB1*15:01, DRB3*01:01, DRB3*02:02, DRB4*01:01, DRB5*01:01. In this study, the IEDB recommended method was utilized to find out the high-ranked epitopes (Wang et al. 2008). 


\section{Prediction of CTL epitopes}

CTL (cytotoxic T-cell) epitopes were identified using CTLpred (http://crdd.osdd.net/raghava/ctlpred/applying/). CTLpred is an online server that utilizes different methods such as quantitative matrix $(\mathrm{QM})$ and machine learning techniques, namely artificial neural network (ANN) and support vector machine (SVM). The accuracy of QM, ANN, and SVM approaches are similar $(70.0 \%$ for QM, $72.2 \%$ for ANN, and $75.2 \%$ for SVM method) (Soria-Guerra et al. 2015). The sensitivity and specificity of QM, ANN, and SVM methods are $0.00,0.51$, and 0.36 , respectively.

\section{Population coverage analysis}

Population coverage for each individual epitope was predicted by the IEDB population coverage analysis resource (http:// tools.iedb.org/population/) against the whole world. This server utilizes LA genotypic frequencies to identify the fraction of individuals that respond to a specific epitope (Bui et al. 2006).

\section{Linear and conformational B-cell epitopes prediction}

Linear or continuous B-cell epitopes were predicted using the BCPRED server (http://ailab.ist.psu.edu/bcpred/predict.html). The server accuracy for the prediction of continuous B-cell epitopes is $74.57 \%$. Predicted regions above the threshold value ( 2 to 2.5 ) are considered to be potential B-cell epitopes (EL-Manzalawy et al. 2008).

Conformational B-cell epitopes were identified from the $3 \mathrm{D}$ structure of the final construct by the DiscoTope 2.0 server at http://www.cbs.dtu.dk/services/DiscoTope/. This server employs a new epitope propensity amino acid score for predicting epitopes (Kringelum et al. 2012). The default threshold, sensitivity, and specificity were used, which were $-3.7,0.47$, and 0.75 , respectively.

\section{Prediction of interferon-gamma inducing epitopes}

To predict interferon-gamma inducing epitopes, the IFNepitope server was utilized at http://crdd.osdd.net/ raghava/ifnepitope. The server uses various methods for identifying interferon-gamma epitopes, including machine learning methods, motifs-based search, and a hybrid approach. The best predicted method is based on the hybrid approach and achieves maximum accuracy of $81.39 \%$ (Dhanda et al. 2013).

\section{Epitope selection and construction of the construct}

The overlapping regions between different predicted epitopes were compared. Several high-ranked and shared parts were selected as the final epitopes from a pool of epitopes. Afterwards, the whole vaccine construct was built by joining the selected epitopes to each other using linkers. A TLR3 agonist was also added to the $\mathrm{N}$-terminal of the construct.

\section{Evaluation of allergenicity and antigenicity}

The allergenicity of the designed multi-epitope peptide vaccine was determined using two various servers, namely AlgPred and AllerTOP 2.0 (http://www.ddgpharmfac.net/ AllerTOP). The AlgPred server (http://www.imtech.res.in/ raghava/algpred) employs six different methods for the prediction of allergens based on the similarity of the known epitopes with any region of proteins. In the present study, the hybrid prediction approach with the highest accuracy was applied as the allergen prediction method (Saha and Raghava 2006). The AllerTOP uses a training set containing 2210 known allergens and 2210 non-allergens of different organisms. The server predicts allergenicity by $\mathrm{k}$ nearest neighbors $(\mathrm{kNN}$ ) algorithm with $85.3 \%$ accuracy (Dimitrov et al. 2014). VaxiJen v2.0 (http://www.ddg-pharmfac.net/ vaxijen/VaxiJen/VaxiJen.html) was employed for prediction of the antigenicity. VaxiJen v2.0 predicts antigenicity based on the principal chemical features of proteins using an alignment-independent approach for various target organisms (bacteria, virus, tumor, parasite, and fungi) with $70-89 \%$ accuracy (Doytchinova and Flower 2007). In this study, "virus" was chosen as the target organism (threshold 0.4).

\section{Evaluation of physicochemical parameters}

To determine different physicochemical properties of the designed multi-epitope peptide vaccine, the ProtParam server at http://web.expasy.org/protparam was employed. Accordingly, theoretical pI (isoelectric point), amino acid composition, instability index, aliphatic index, molecular weight (MW), in vitro and in vivo half-life, and grand average of hydropathicity (GRAVY) were calculated (Gasteiger et al. 2005).

\section{Homology modeling}

The 3D structure of the designed construct was predicted using I-TASSER at http://zhanglab.ccmb.med.umich.edu/ITASSER. The server works based on iterative threading assembly simulations. The $3 \mathrm{D}$ structure modeling of the peptide construct by the I-TASSER consists of several steps: (1) prediction of threading templates, (2) simulation of iterative structure assembly, (3) selection and refinement of the predicted model, and (4) structure-based function annotations (Yang et al. 2015). The quality of the predicted 3D models by I-TASSER is estimated by a C-score (confidence score). A 
model with a higher $\mathrm{C}$-score poses more confidence (Yang and Zhang 2015).

\section{Refinement of the tertiary modeled structure}

The refinement process for the predicted 3D structure was done by GalaxyRefine. The best 3D model obtained from ITASSER based the $\mathrm{C}$-score was introduced to the GalaxyRefine server at http://galaxy.seoklab.org/cgi-bin/ submit.cgi?type=REFINE. In this server, mild and aggressive relaxation approaches are employed for refinement of the designed protein (Heo et al. 2013). Both the backbone and side-chain structure qualities are enhanced by repetitive perturbation and relaxation. The server improves the initial models with a high probability $(>50 \%$ ) (Shin et al. 2014).

\section{Validation of the refined tertiary structure}

To recognize potential errors in the predicted 3D model, the refined structures were evaluated by various servers, including RAMPAGE (Ramachandran Plot Assessment), ProSA-web (Protein Structure Analysis), and verify 3D. The RAMPAGE server at http://mordred.bioc.cam.ac.uk/ rapper/ rampage.php estimates phi-psi torsion angles for each residue and classifies them in favored, allowed, and outlier groups (Lovell et al. 2003). The verify 3D at http://services.mbi. ucla.edu/Verify_3D/ compares the predicted model to its residues by assigning a structural class based on its location (Luthy et al. 1992). The ProSA-web at (https://prosa.services. came.sbg.ac.at/prosa.php) estimates an overall quality score for a specific input structure. The server requires only $\mathrm{C} \alpha$ atoms so that low-resolution structures can be evaluated. The interaction energy of each residue with the rest of the structure is analyzed and potential errors in the protein structure are calculated (Wiederstein and Sippl 2007). In this study, discovery studio 3.5 and PyMOL programs were employed for visualization of $3 \mathrm{D}$ models.

\section{Molecular docking of the peptide vaccine and TLR3}

The Cluspro 2.0 server at http://cluspro.bu.edu/login.php was used for the molecular docking analysis of the designed vaccine as the ligand and the TLR3 as the receptor. This fast rigid-body protein-protein docking server performs docking in three general steps. The first step is rigid-body docking using PIPER, which is based on the fast Fourier transform (FFT) program. The second step is isolating highly populated low energy (clustering) by the pairwise root mean square deviation (RMSD). Finally, the third step is a refinement process via the medium-range optimization method of SDU (Semidefinite Programming-Based Underestimation) (Kozakov et al. 2010).

\section{Codon optimization and in silico cloning}

Reverse translation and codon optimization were performed using Codon Usage Wrangler (http://www.mrclmb.cam.ac. $\mathrm{uk} / \mathrm{ms} / \mathrm{methods} /$ codon.html) and the GenScript Rare Codon Analysis tool (https://www.genscript.com/tools/rare-codonanalysis), respectively. Codon adaptation index (CAI), GC content, and codon frequency distribution (CFD) were evaluated using the GenScript Rare Codon Analysis. These key parameters influence the protein expression level in the Eschercia coli host. To prepare the adopted sequences for cloning in E. coli, pET-14b vector was chosen and NdeI and $\mathrm{BamHI}$ restriction sites were added to the $\mathrm{N}$ and $\mathrm{C}$-terminal of the final construct, respectively.

\section{Results}

\section{Identification of immunodominant epitopes and population coverage analysis}

The highly-scored epitopes over the 38 most frequent HLA class I and II alleles were identified using IEDB, and the selected segments were compared to each other to find the overlap regions as the high-ranked epitopes (Table S1 and S2). Subsequently, the overlapping regions obtained from the HLA class I and II binding results were compared with the high-ranked results of CTLPred, and their shared regions were exploited (Table S3). Three linear B-cell epitopes were identified by BCPRED, as listed in Table 1. Using DiscoTope server, five conformational B-cell epitopes were predicted in the final 3D model out of a total of 157 residues (Table 1). The population coverage percentages for each epitope and their epitope sets were calculated by the IEDB server (Tables S1, S2, and S3). The epitopes with a higher global population coverage were carefully selected for the final construct to ensure achieving a universal multi-epitope peptide vaccine. Based on the high-ranked and shared regions, six epitopes were selected from the four antigenic proteins (Table 2). The selected epitopes of each protein were fused together by the AAYKK linker. Then, these segments were joined to each other using the GSGSGS linker. LL37 was added as an adjuvant to the $\mathrm{N}$-terminal of the construct using the EAAAK linker. The final designed construct consisted of 157 amino acid residues is illustrated in Fig. 1. Using IFNepitope server, two segments were found as IFN-gamma inducing epitopes on the final vaccine construct (Table 3 ).

\section{Evaluation of allergenicity, antigenicity, and physicochemical parameters}

The antigenicity of the whole construct was $0.2197 \%$. Based on the obtained results, the peptide vaccine could trigger efficient 
Table 1 Linear and conformational B-cell epitopes determined by BCPREDS and DiscoTope servers

\begin{tabular}{|c|c|c|c|c|}
\hline \multicolumn{5}{|c|}{ Linear B-cell epitopes } \\
\hline \multicolumn{3}{|l|}{ Position } & \multicolumn{2}{|l|}{ Epitopes } \\
\hline \multicolumn{2}{|l|}{$7-17$} & & \multicolumn{2}{|l|}{ RKSKEKIGKEF } \\
\hline \multicolumn{2}{|l|}{$90-100$} & & \multicolumn{2}{|l|}{ GSGSGSANRNR } \\
\hline \multicolumn{2}{|l|}{$134-147$} & & \multicolumn{2}{|l|}{ KTFPPTEPKGSGSG } \\
\hline \multicolumn{5}{|c|}{ Conformational B-cell epitopes } \\
\hline Residuenumber & Aminoacid & Contactnumber & Propensity score & DiscoTope score \\
\hline 4 & ASP & 2 & -3.408 & -3.246 \\
\hline $7-15$ & $\begin{array}{l}\text { ARG, LYS,SER, LYS,GLU, LYS, ILE, } \\
\text { GLY,LYS }\end{array}$ & $5,0,0$ & -3.163 & -3.029 \\
\hline 18 & LYS & 7 & -2.744 & -3.233 \\
\hline 140 & GLU & 2 & -3.753 & -3.55 \\
\hline $142-146$ & LYS, GLY, SER, GLY, SER & $4,16,6,0,0$ & $\begin{array}{l}-2.254,-2.061,-2.620 \\
\quad-2.861,-3.228\end{array}$ & $\begin{array}{l}-2.455,-3.664,-3.009 \\
-2.532,-2.857\end{array}$ \\
\hline
\end{tabular}

immunity against the pathogen. Furthermore, the designed construct did not induce allergen-specific antibodies. The physicochemical properties of the multi-epitope peptide vaccine indicated that the theoretical pI of the protein was 10.26. Moreover, the MW of the protein was $17.4 \mathrm{kDa}$. The total numbers of positively and negatively charged residues were 25 and 9, respectively. The estimated half-lives were computed to be $5.5 \mathrm{~h}$ (in mammalian reticulocytes, in vitro). The instability index was calculated as 36.43. A protein whose instability index is smaller than 40 is considered as a stable protein. Therefore, the designed peptide vaccine was sTable The aliphatic index was determined as 79.68. The high aliphatic index illustrated the high-protein stability in a wide range of temperatures. Moreover, the GRAVY value of the peptide construct was -0.234 . A negative GRAVY value indicates that the protein is hydrophilic and has an appropriate interaction in an aqueous environment.

\section{Homology modeling, refinement, and molecular docking of the peptide vaccine}

Model 1 was selected as the best model with the highest $\mathrm{C}$ score value (-2.06). I-TASSER introduces the top five models based on the C-score. The C-score is in the range of [-5 to 2], and a model with higher confidence has a higher $\mathrm{C}$-score value.

GalaxyRefine introduced five 3D refined models. All obtained models were validated and a high-quality $3 \mathrm{D}$ model according to z-score, potential errors, and the overall quality factor (obtained in the next step) was selected. The initial and refined 3D models are compared in Fig. 2. The initial best model of the designed peptide was evaluated for potential errors. Ramachandran analysis was performed before and after refinement processes. In the initial model, $76.1 \%, 20.6 \%$, and $3.2 \%$ of residues were found in the favored, allowed, and outlier regions, respectively. While in the refined model, 92.9\%, 5.8\%, and $1.3 \%$ of residues were located in the favored, allowed, and outlier regions, respectively.

The Z-score of the initial input model and refined model showing the quality of the best model calculated by ProSA-web, were (-6.28) and (-6.25), respectively (Fig. S1a and Fig. S1b). These scores were similar to each other and were within the range of scores typically found in the native proteins of similar sizes. The Verify $3 \mathrm{D}$ score indicated that in the initial model, $76.43 \%$ of residues had an average $3 \mathrm{D}-1 \mathrm{D}$ score greater than 0.2 (Fig. 4a). In the refined model, the Verify 3D score was improved to $80.89 \%$. It means that more amino acid residues were located in an acceptable side chain environment (Fig. S2a). In molecular docking, ten models were generated based on the biophysical properties of the designed vaccine as the ligand and TLR3 as the receptor, using the ClusPro server. Finally, the most plausible docked model structure was selected based on the interaction regions (Fig. 3). PDB formats of the docked structures were visualized using discovery studio 3.5 and the PyMOL software.

\section{Codon optimization and in silico cloning}

CAI of the optimized sequence was 1.0. It is considered ideal for expression in $E$. coli (ideal value for CAI is between 0.8 and 1.0 (Fig. 4a). The average GC content of the DNA sequence was $54.21 \%$ (Fig. 4b). The ideal range of GC content is between 30 and $70 \%$. Any region outside of this range will decrease protein expression. The CFD value was $100 \%$ for the optimized sequence. A CFD lower than 30 will negatively affect translational efficiency (Fig. 4c). These results proposed that the optimized sequence could achieve an acceptable expression in E. coli. 
Table 2 The six final epitope segments that were selected based on the results obtained surveying four antigenic viral proteins using several servers

\begin{tabular}{|c|c|c|c|c|}
\hline Protein & Start-End position & Sequence & Length of sequence & Alleles \\
\hline S Protein & $\begin{array}{l}231-259 \\
341-353\end{array}$ & $\begin{array}{l}\text { IGINITRFQTLLALHRSYLTPGDSSSGWT } \\
\text { VFNATRFASVYAW }\end{array}$ & $\begin{array}{l}29 \\
13\end{array}$ & $\begin{array}{l}\text { HLA-B*08:01 } \\
\text { HLA-B*14:02 } \\
\text { HLA-B*27:02 } \\
\text { HLA-B*27:05 } \\
\text { HLA-B*40:01 } \\
\text { HLA-A*02:01 } \\
\text { HLA-A*24:02 } \\
\text { HLA-A*31:01 } \\
\text { HLA-A*68:01 } \\
\text { HLA-A*23:01 } \\
\text { HLA-B*08:01 } \\
\text { HLA-B*40:01 } \\
\text { HLA-B*44:02 } \\
\text { HLA-B*44:03 }\end{array}$ \\
\hline M Protein & $\begin{array}{l}40-49 \\
95-102\end{array}$ & $\begin{array}{l}\text { ANRNRFLYII } \\
\text { YFIASFRL }\end{array}$ & $\begin{array}{l}10 \\
8\end{array}$ & $\begin{array}{l}\text { HLA-A*01:01 } \\
\text { HLA-A*02:01 } \\
\text { HLA-A*11:01 } \\
\text { HLA-A*23:01 } \\
\text { HLA-A*24:02 } \\
\text { HLA-B*08:01 } \\
\text { HLA-B*14:02 } \\
\text { HLA-B*27:02 } \\
\text { HLA-B*27:05 } \\
\text { HLA-B*44:02 } \\
\text { HLA-B*35:01 } \\
\text { HLA-A*31:01 } \\
\text { HLA-A*68:01 } \\
\text { HLA-A*23:01 } \\
\text { HLA-B*07:01 } \\
\text { HLA-B*08:01 }\end{array}$ \\
\hline N Protein & $352-369$ & LLNKHIDAYKTFPPTEPK & 18 & $\begin{array}{l}\text { HLA-A*03:01 } \\
\text { HLA-A*11:01 } \\
\text { HLA-A*23:01 } \\
\text { HLA-B*27:02 } \\
\text { HLA-A*31:01 } \\
\text { HLA-A*68:01 } \\
\text { HLA-DRB1*09:01 } \\
\text { HLA-DRB1*12:01 } \\
\text { HLA-DRB1*13:02 } \\
\text { HLA-DRB1*13:03 } \\
\text { HLA-DRB1*14:01 } \\
\text { HLA-DRB1*15:01 }\end{array}$ \\
\hline ORF3a & $103-111$ & APFLYLYAL & 9 & $\begin{array}{l}\text { HLA-A*01:01 } \\
\text { HLA-A*02:01 } \\
\text { HLA-A*03:01 } \\
\text { HLA-A*23:01 } \\
\text { HLA-B*07:02 } \\
\text { HLA-B*08:01 } \\
\text { HLA-B*27:02 } \\
\text { HLA-B*35:01 } \\
\text { HLA-B*44:02 } \\
\text { HLA-B*44:03 } \\
\text { HLA-DRB1*03:01 } \\
\text { HLA-DRB1*07:01 } \\
\text { HLA-DRB1*08:02 } \\
\text { HLA-DRB1*09:01 } \\
\text { HLA-DRB1*11:01 } \\
\text { HLA-DRB1*11:06 } \\
\text { HLA-DRB1*13:02 } \\
\text { HLA-DRB1*13:03 } \\
\text { HLA-DRB1*14:01 }\end{array}$ \\
\hline
\end{tabular}




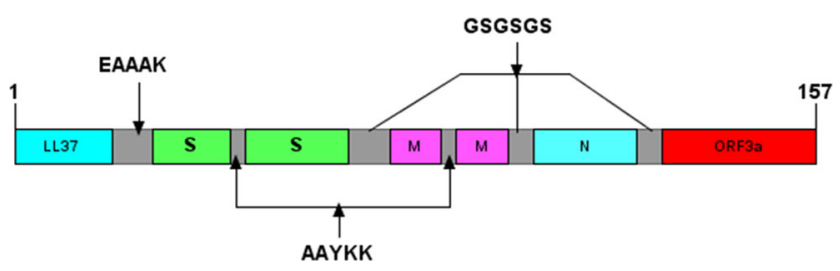

Fig. 1 Schematic diagram of the final multi-epitope polypeptide vaccine construct. The sequence consists of 157 residues. The first 37 amino acids are related to LL37 adjuvant, followed by the six immunodominant epitopes from S (spike), M (membrane), N (nucleocapsid), and ORF (open reading frame) 3 a proteins linked together by AAYKK and GSGSGS linkers

\section{Discussion}

The advances made in bioinformatics and novel computational and predictive tools have greatly contributed to the development of novel vaccines. Moreover, further understanding of the immune system's regulatory and functional pathways has proven to improve the development of effective and safe vaccine candidates (Negahdaripour et al. 2018). The current pandemic outbreak of SARS-CoV-2 led to worldwide collaborations in the urgent searches for preventive and therapeutic solutions. Although development of numerous vaccine candidates worldwide is proceeding with unprecedented speed, some vaccines are still in evaluation phases (Krammer 2020). While the health authority approval in different countries has been announced for some vaccines including those developed by Moderna (Jackson et al. 2020; Mahase 2020), Pfizer and BioNTech (Walsh et al. 2020), Janssen, and AstraZeneca and the University of Oxford (Ramasamy et al. 2021), as well as Sputnik V, Sinopharm vaccine, and some others. However, there are several concerns regarding the storage, cold chain transfer conditions, and costs of some of these pioneer vaccines. Moreover, availability is still an issue, which might limit their administration, especially in the developing countries (Ashok et al. 2017). Multi-epitope polypeptide vaccines could be attractive alternatives considering their safety, more convenient development and storage, and less production costs. Their platform also allows inclusion of several different epitopes that can induce both cellular and humoral immune responses (Negahdaripour et al. 2017b). Furthermore, in silico approaches for designing such vaccines could save

Table 3 Predicted IFN-gamma inducing epitopes in the final vaccine construct via IFNepitope sever

\begin{tabular}{lll}
\hline Start- & Sequence & Score \\
End & & \\
\hline $352-369$ & LLNKHIDAYKTFPPTEPK & 0.064 \\
$103-111$ & APFLYLYAL & 0.394 \\
\hline
\end{tabular}

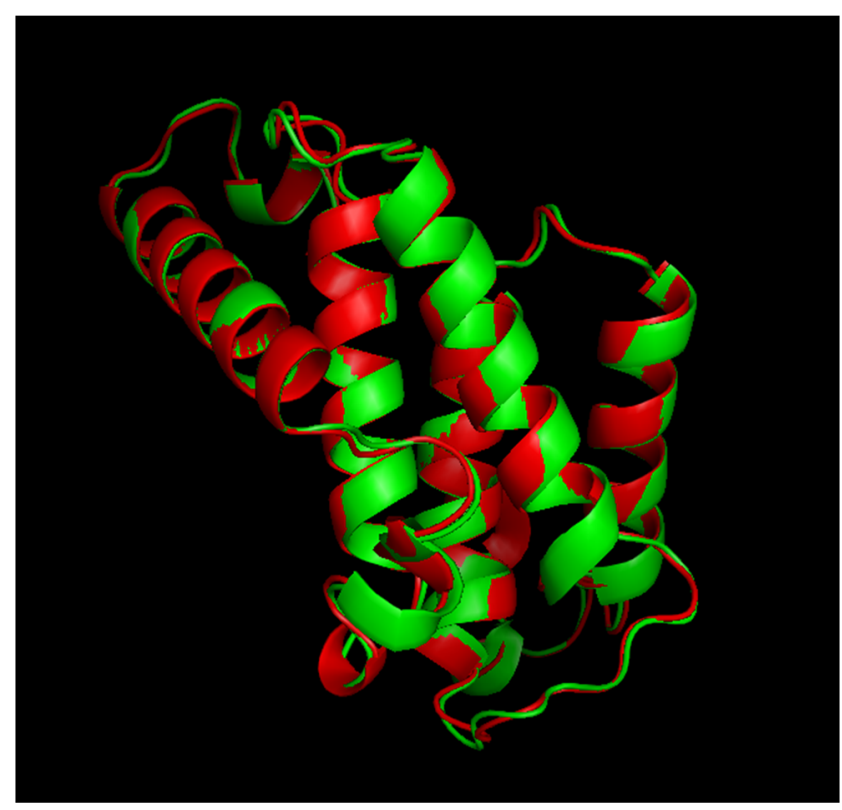

Fig. 2 Superimposition of the initial (red) and final (green) 3D multiepitope peptide vaccine structures, before and after structure refinement

considerable amounts of time, energy, and costs, which facilitates vaccine production process (O'Hagan and De Gregorio 2009).

In the worldwide struggle for finding solutions to help prevent further loss due to COVID-19, several bioinformatics studies have been carried out to design vaccine candidates for potential clinical studies. For instance, in two computational vaccine designing studies, only the $\mathrm{S}$ protein was employed for epitope detection (Samad et al. 2020, Yang et al. 2021). In another in silico study, designing a vaccine construct out of multiple $\mathrm{S}$ protein epitopes were performed to induce both CD4 and CD8 T-cell response against SARS-CoV-2 (Abraham Peele et al. 2020). Dong et al. developed a multiepitope protein vaccine consisting of epitopes from nine different proteins of SARS-CoV-2 (ORF7a protein, ORF8 protein, nsp (nonstructural protein) 9, nsp6, nsp3, endoRNAse, ORF3a protein, membrane glycoprotein, and nucleocapsid phosphoprotein) utilizing bioinformatics and computational tools (Dong et al. 2020). Besides, a reverse vaccinology in silico study employed the viral $\mathrm{N}$ protein, ORF3a, and $\mathrm{M}$ protein to achieve a multi-epitope vaccine construct (Enayatkhani et al. 2020). In this study, various epitopes found on $\mathrm{S}, \mathrm{N}$, and $\mathrm{M}$ proteins along with ORF3a were employed simultaneously as a novel approach for designing a multi-epitope vaccine against SARS-CoV-2 to potentially enhance its antigenicity and efficacy. $\mathrm{N}$-glycans present in the structure of viral S protein, play a crucial role in modulation of RBD conformational dynamics thereby facilitating ACE2 receptor recognition by the $\mathrm{S}$ protein (Casalino et al. 2020). The SARS-CoV-2 N protein, which takes part in RNA packaging processes, also possesses immunological value, since immunological assays provided data that showed the presence of antibodies against the $\mathrm{N}$ protein (Zeng et al. 2020). The viral 
a

Codon Adaptation Index (CAI)

Escherichia

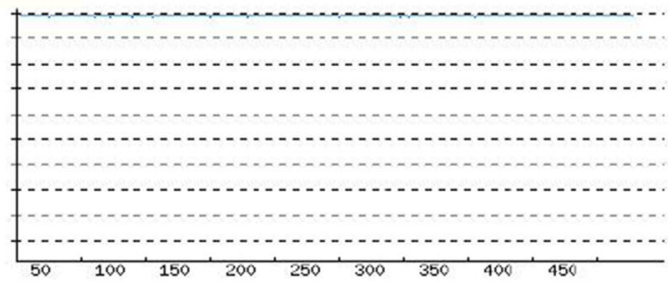

CAI: 1.00

b

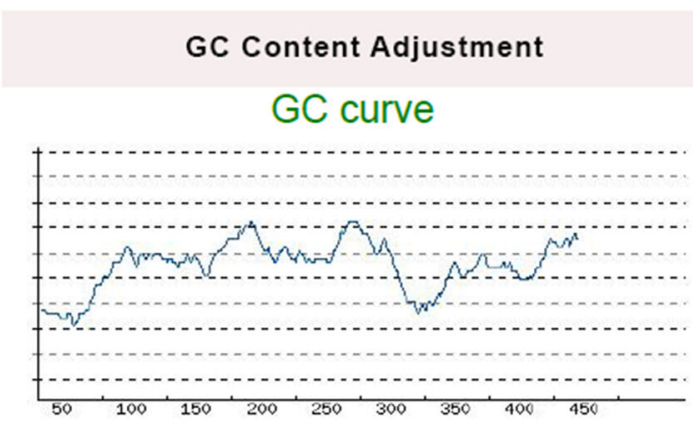

Average GC content: $54.21 \%$

c

Codon Frequency Distribution (CFD)

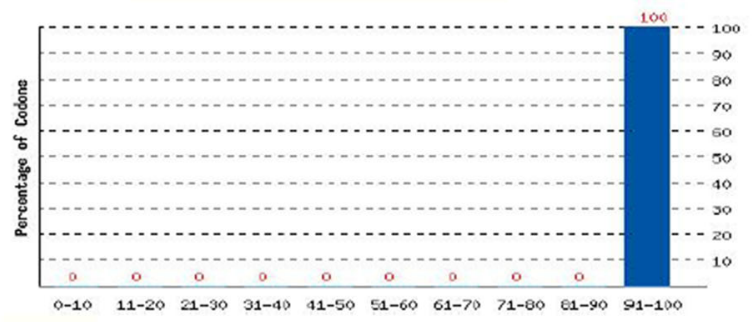

Escherichia

Fig. 4 Codon adaptation index (CAI), GC content, and codon frequency distribution (CFD) of the reverse translated gene for cloning of the construct, evaluated by the GenScript Rare Codon Analysis tool. (a) The CAI of the optimized sequence was 1.0, which is ideal for expression in E. coli. (b) The average GC content of the DNA sequence was $54.21 \%$, which indicates proper protein expression. (c) The CFD value was $100 \%$ for the optimized sequence, which suggests an optimum translational efficiency. These results proposed that the optimized sequence could achieve an acceptable expression in E. coli

M protein also participates in virus assembly (Masters 2006). As indicated in previous studies, ORF3a of SARS-CoV is a transmembrane protein consisting of various motifs that provide proapoptotic functions (Chan et al. 2009). Moreover, a recent study carried out in China pointed out the structural similarities between ORF3a proteins of SARS-CoV and SARS-CoV-2 and indicated that the ORF3a of SARS-CoV-2 also induced cellular

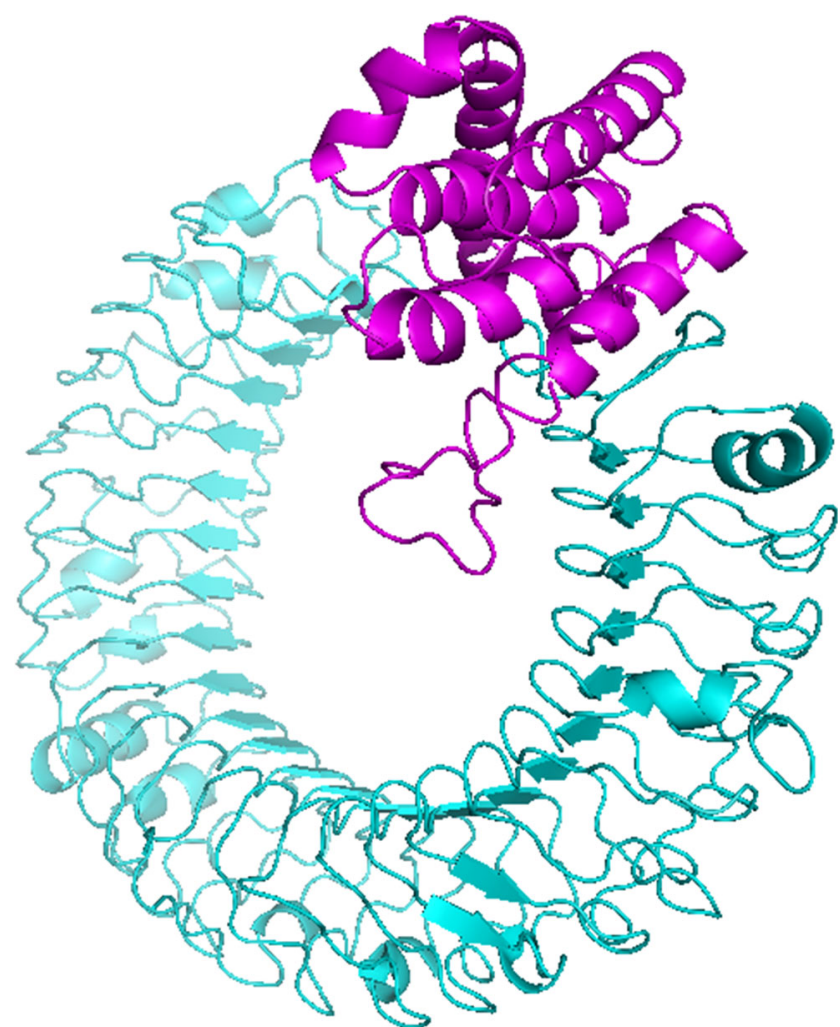

Fig. 3 The docked model (cartoon representation) of the multi-epitope peptide vaccine and TLR3 obtained by the Cluspro 2.0 server. TLR3 is shown in cyan and the designed vaccine is in magenta colors. It indicated a significant affinity between the designed vaccine and TLR3

apoptosis (Ren et al. 2020). It was shown that ORF3a accessory protein stimulates signals for NLRP3 (NOD- [nucleotide-binding domain], LRR- [leucine-rich repeat], and pyrin domaincontaining protein 3 ) inflammasome activation and induces the production of IL-1 $\beta$ through induction of the pro-IL-1 $\beta$ gene (Siu et al. 2019). IL-1 $\beta$, a critical pro-inflammatory cytokine, is secreted in the early stages of the viral infection (De Lang et al. 2007). Furthermore, ORF3a induces in vitro neutralizing antibodies against SARS-CoV-2 (Qiu et al. 2005). Hence, we assumed that using the epitopes on four different viral proteins that are important in pathogenesis could not only grant the vaccine more antigenicity, but also help the vaccine to maintain its efficiency even in the case of viral mutations. Obviously, SARSCoV-2 as an RNA virus bears a high chance of mutations. Given that mutations may not occur in all the viral proteins at the same time, this is a very useful logical strategy for achieving an efficient vaccine.

In this study, overlapping epitopes were selected from different immunological classes of B-cell and T-cell stimulating antigens through using various servers. It was found that the combination of several promising epitopes in a single peptide construct helps address the low immunogenicity problem associated with peptide vaccines (Abraham et al. 2020, Livingston et al. 2002). Hence, in addition to employment of 
several T- and B-cell inducing epitopes, the presence of IFNgamma inducing epitopes was also affirmed. Some cytokines, especially IFN-gamma, play an important role in mediating protection (Samuel 2001).

Moreover, addition of LL37 (residues 17-29), as a TLR3 agonist adjuvant, could reinforce its ability in stimulation of innate immune responses leading to more potent adaptive immune responses. An increasing trend is found in using TLR agonists as potential built-in adjuvants for the development of novel vaccines. TLRs, as a family of pattern recognition receptors (PRRs), recognize conserved pathogenic structures, such as pathogen-associated molecular patterns (PAMPs) (Suresh and Mosser, 2013), and expedite antigen presentation on the cell surface, thereby further promoting the immune response to the vaccine construct (Moyle and Toth, 2013). A number of TLR ligands, such as TLR3, TLR4, TLR5, and TLR9 (Reed et al. 2016), have been identified as adjuvants, which could enhance CTL response via CD4 + T-helper cells (van der Burg et al. 2006). TLR signaling could be carried out by five different adapter proteins (MyD88, MAL, TRIF, TRAM, and SARM). In this regard, TLR3 only signals through TRIF and TRAM protein adapters (O'Neill and Bowie, 2007).

In regards to adjuvant, a TLR-4 agonist, $50 \mathrm{~S}$ ribosomal protein L2 was used in a vaccine designed against SARSCoV-2 (Yang et al. 2021), while $50 \mathrm{~S}$ ribosomal protein L7/ L12, was employed for the same purpose in another study (Samad et al. 2020). Selection of LL37 as a TLR3 agonist in our study was based on the following rational findings about role of TLR3 in coronaviruses and the nature of LL37. It was confirmed that TLR3 is able to induce strong immune responses against viruses, especially MERS-CoV (Middle East respiratory syndrome coronavirus) and SARS-CoV (Lester and Li 2014). LL37 is an antimicrobial protein with the ability to modulate dendritic cell (DC) activity and also chemotactic properties. LL37 could amplify antigen-specific immune responses (An et al. 2005; Kim et al. 2015) by facilitating viral responses through the activation of TLR3 signaling (Lai et al. 2011). Accordingly, LL37 was incorporated into the designed vaccine sequence to improve the immunogenicity of the construct.

For joining different segments of the vaccine construct, several linkers, namely GSGSGS, AYYKK, and EAAAK, were used. Linkers facilitate antigen processing and avoid formation of the "junctional epitopes", which could alter the structure of the peptide vaccine (Livingston et al. 2002). The GSGSGS linker allows relatively free rotation of the segments in the vaccine construct. Small amino acids such as glycine and serine provide flexibility for the peptide vaccine (Negahdaripour et al. 2017a). The KKAAY linker increases the probability of epitope presentation in both MHC I and II pathway (Vakili et al. 2018), and EAAAK is a rigid linker that causes a more effective separation between the functional domains (Arai et al. 2004). Thus, these different linkers were employed in various parts of the designed structure. Such linkers are usually employed in different computationally designed vaccines (Hajighahramani et al. 2019, Samad et al. 2020, Yang et al. 2021).

Our designed candidate vaccine comprised of 157 amino acids, which possessed acceptable immunological and structural properties according to the physicochemical results. The length of multi-epitope peptide vaccines could be very different mostly based on the choice of the designers. For example, multi-epitope SARS-CoV-2 vaccines designed by Dong et al. and Yang et al. had a length of 864 and 649 amino acids, respectively (Dong et al. 2020, Yang et al. 2021). Of note, some considerations in respect to convenient cloning, less possibility of conformational instability and huge variations, and formulation concerns should be taken into account in this regard.

The instability index, half-life, and theoretical PI revealed that the peptide vaccine was sTable Furthermore, the construct was soluble, immunogen, and nonallergic (as mentioned in Section 3.2), which means that the designed peptide could be proposed as an appropriate vaccine candidate.

The model refinement and validation of the initial tertiary structure of the vaccine helped to improve the quality of the refined model remarkably. Additionally, results of the docking study, as seen in Fig. 3, indicated that the designed vaccine had a significant affinity towards the TLR3.

Finally, the CAI and GC content of the codon optimized gene sequence suggested ideal expression of the recombinant protein in E. coli host.

All in all, the proposed multi-epitope peptide vaccine is expected to induce robust humoral and cellular immune responses against the virus due to all the mentioned acceptable immunological, structural, and physicochemical properties. Selecting epitopes from three viral structural proteins and another functional protein suggests the vaccine's protective ability irrespective of probable virus mutations. However, this candidate vaccine construct should be further investigated in a course of various studies including in vitro, in vivo, and clinical trials, respectively, to confirm its efficacy and safety. Application of carriers could also further improve its antigenicity in future evaluations.

Abbreviations ACE2, Angiotensin converting enzyme-2; ANN, Artificial neural network; CAI, Codon adaptation index; CFD, Codon frequency distribution; COVID-19, Coronavirus disease 2019; C-score, Confidence score; CTL, Cytotoxic T-cell; DC, Dendritic cell; E, Envelope protein; EUA, Emergency use authorization; FDA, Food and Drug Administration; FFT, Fast fourier transform; GRAVY, Grand average of hydropathicity; HLA, Human leukocyte antigen; IEDB, Immune epitope databases; IFN, Interferon; IL, Interleukin; kNN, K nearest neighbors; LRR, Leucine-rich repeat; M, Membrane protein; MAL, MyD88adaptor-like protein; MERS-CoV, Middle East respiratory syndrome coronavirus; MHC, Major histocompatibility complex; MyD88, Myeloid 
differentiation primary response 88; MW, Molecular weight; N, Nucleocapsid protein; NCBI, National Centre for Biotechnology Information; NLRP3, NOD-, LRR- and pyrin domain-containing protein 3; NOD, Nucleotide-binding domain; nsp, Nonstructural protein; ORF, Open reading frame; PAMPs, Pathogen-associated molecular patterns; PRRs, Pattern recognition receptors; pI, isoelectric point; ProSA-web, Protein Structure Analysis; QM, Quantitative matrix; RAMPAGE, Ramachandran Plot Assessment; RBD, Receptor binding domain; RMSD, Root mean square deviation; S, Spike protein; SARM, Sterile a- and armadillo-motif-containing protein; SARS-CoV-2, Severe acute respiratory syndrome coronavirus-2; SDU, Semidefinite ProgrammingBased Underestimation; TLR, Support vector machine (SVM); Toll-like receptor; TRIF, TIR-domain-containing adaptor protein inducing IFN- $\beta$; TRAM, TRIF-related adaptor molecule

Supplementary Information The online version contains supplementary material available at https://doi.org/10.1007/s11756-021-00866-y.

Funding This study was supported by Grant No. 98-01-106-22099 from the Research Council of Shiraz University of Medical Sciences, Shiraz, Iran.

Data availability More data are available as supplementary materials.

\section{Declarations}

Conflict of interest The authors declare no conflicts of interest.

Ethical approval The approval code of IR.SUMS.REC.1399.056 is dedicated to this project by Shiraz University of Medical Sciences.

Informed consent Not applicable, since no patient was investigated in this study.

\section{References}

Abraham Peele K, Srihansa T, Krupanidhi S, Ayyagari VS, Venkateswarulu TC (2020) Design of multi-epitope vaccine candidate against SARS-CoV-2: a in-silico study. J Biomol Struct Dyn:19. https://doi.org/10.1080/07391102.2020.1770127

An LL, Yang YH, Ma XT, Lin YM, Li G, Song YH, Wu KF (2005) LL37 enhances adaptive antitumor immune response in a murine model when genetically fused with M-CSFRJ6-1 DNA vaccine. Leuk Res 29:535-543. https://doi.org/10.1016/j.leukres.2004.11.009

Arai R, Wriggers W, Nishikawa Y, Nagamune T, Fujisawa T (2004) Conformations of variably linked chimeric proteins evaluated by synchrotron X-ray small-angle scattering. Proteins 57:829-838. https://doi.org/10.1002/prot.20244

Ashok A, Brison M, LeTallec Y (2017) Improving cold chain systems: Challenges and solutions. Vaccine 35(17):2217-2223. https://doi. org/10.1016/j.vaccine.2016.08.045

Bagheri A, Moezzi SMI, Mosaddeghi P, Parashkouhi SN, Hoseini SMF, Badakhshan F, Negahdaripour M (2020) Interferon-inducer antivirals: potential candidates to combat COVID-19. Int Immunopharmacol:107245. https://doi.org/10.1016/j.intimp.2020. 107245

Bui HH, Sidney J, Dinh K, Southwood S, Newman MJ, Sette A (2006) Predicting population coverage of T-cell epitope-based diagnostics and vaccines. BMC Bioinform 7:153. https://doi.org/10.1186/14712105-7-153

Casalino L, Gaieb Z, Goldsmith JA, Hjorth CK, Dommer AC, Harbison AM, Fogarty CA, Barros EP, Taylor BC, McLellan JS (2020)
Beyond shielding: the roles of glycans in the SARS-CoV-2 spike protein. ACS Cent Sci 6:1722-1734. https://doi.org/10.1021/ acscentsci.0c01056

Chan CM, Tsoi H, Chan WM, Zhai S, Wong CO, Yao X, Chan WY, Tsui SKW, Chan HYE (2009) The ion channel activity of the SARScoronavirus 3 a protein is linked to its pro-apoptotic function. Int $\mathrm{J}$ Bioch Cell Biol 41:2232-2239. https://doi.org/10.1016/j.biocel. 2009.04.019

Coffman RL, Sher A, Seder RA (2010) Vaccine adjuvants: Putting innate immunity to work. Immunity 33(4):492-503. https://doi.org/10. 1016/j.immuni.2010.10.002

De Lang A, Baas T, Teal T, Leijten LM, Rain B, Osterhaus AD, Haagmans BL, Katze MG (2007) Functional genomics highlights differential induction of antiviral pathways in the lungs of SARSCoV-infected macaques. PLOS Pathog 3:e112. https://doi.org/10. 1371/journal.ppat.0030112

Dhanda SK, Vir P, Raghava GP (2013) Designing of interferon-gamma inducing MHC class-II binders. Biol Direct 8:30. https://doi.org/10. 1186/1745-6150-8-30

Dimitrov I, Bangov I, Flower DR, Doytchinova I (2014) AllerTOP v.2-a server for in silico prediction of allergens. J Mol Model 20:2278. https://doi.org/10.1007/s00894-014-2278-5

Dong R, Chu Z, Yu F, Zha Y (2020) Contriving multi-epitope subunit of vaccine for COVID-19: immunoinformatics approaches. Front Immunol 11:1784. https://doi.org/10.3389/fimmu.2020.01784

Doytchinova IA, Flower DR (2007) VaxiJen: a server for prediction of protective antigens, tumour antigens and subunit vaccines. BMC Bioinform 8(1):4. https://doi.org/10.1186/1471-2105-8-4

Drosten C, Günther S, Preiser W, van der Werf S, Brodt H-R, Becker S, Rabenau H, Panning M, Kolesnikova L, Fouchier RAM, Berger A, Burguière A-M, Cinatl J, Eickmann M, Escriou N, Grywna K, Kramme S, Manuguerra J-C, Müller S, Rickerts V, Stürmer M, Vieth S, Klenk H-D, Osterhaus ADME, Schmitz H, Doerr HW (2003) Identification of a novel coronavirus in patients with severe acute respiratory syndrome. N Engl J Med 348:1967-1976. https:// doi.org/10.1056/nejmoa030747

EL-Manzalawy Y, Dobbs D, Honavar V (2008) Predicting linear B-cell epitopes using string kernels. J Mol Recognit 21:243-255. https:// doi.org/10.1002/jmr.893

Enayatkhani M, Hasaniazad M, Faezi S, Guklani H, Davoodian P, Ahmadi N, Einakian MA, Karmostaji A, Ahmadi K (2020) Reverse vaccinology approach to design a novel multi-epitope vaccine candidate against COVID-19: an in silico study. J Biomol Struct Dyn:1-16. https://doi.org/10.1080/07391102.2020.1756411

FDA US (2020a) Pfizer-BioNTech COVID-19 vaccine. https://www.fda. gov/emergency-preparedness-and-response/coronavirus-disease2019-covid-19/pfizer-biontech-covid-19-vaccine\#additional. Accessed 22 May 2021

FDA US (2020b) Moderna COVID-19 vaccine. https://www.fda.gov/ emergency-preparedness-and-response/coronavirus-disease-2019covid-19/moderna-covid-19-vaccine. Accessed 22 May 2021

FDA US (2021) Janssen COVID-19 vaccine. https://www.fda.gov/ emergency-preparedness-and-response/coronavirus-disease-2019covid-19/janssen-covid-19-vaccine. Accessed 22 May 2021

Gasteiger E, Hoogland C, Gattiker A, Duvaud Se, Wilkins MR, Appel RD, Bairoch A (2005) Protein identification and analysis tools on the ExPASy server. Methods Mol Biol 112:531-552. https://doi. org/10.1385/1-59259-584-7:531

Gordon DE, Jang GM, Bouhaddou M, Xu J, Obernier K, White KM, O’Meara MJ, Rezelj VV, Guo JZ, Swaney DL, Tummino TA, Hüttenhain R, Kaake RM, Richards AL, Tutuncuoglu B, Foussard H, Batra J, Haas K, Modak M, Kim M, Haas P, Polacco BJ, Braberg H, Fabius JM, Eckhardt M, Soucheray M, Bennett MJ, Cakir M, McGregor MJ, Li Q, Meyer B, Roesch F, Vallet T, Mac Kain A, Miorin L, Moreno E, Naing ZZC, Zhou Y, Peng S, Shi Y, Zhang Z, Shen W, Kirby IT, Melnyk JE, Chorba JS, Lou K, Dai SA, Barrio- 
Hernandez I, Memon D, Hernandez-Armenta C, Lyu J, Mathy CJP, Perica T, Pilla KB, Ganesan SJ, Saltzberg DJ, Rakesh R, Liu X, Rosenthal SB, Calviello L, Venkataramanan S, Liboy-Lugo J, Lin Y, Huang XP, Liu YF, Wankowicz SA, Bohn M, Safari M, Ugur FS, Koh C, Savar NS, Tran QD, Shengjuler D, Fletcher SJ, O'Neal MC, Cai Y, Chang JCJ, Broadhurst DJ, Klippsten S, Sharp PP, Wenzell NA, Kuzuoglu-Ozturk D, Wang HY, Trenker R, Young JM, Cavero DA, Hiatt J, Roth TL, Rathore U, Subramanian A, Noack J, Hubert M, Stroud RM, Frankel AD, Rosenberg OS, Verba KA, Agard DA, Ott M, Emerman M, Jura N, von Zastrow M, Verdin E, Ashworth A, Schwartz O, d'Enfert C, Mukherjee S, Jacobson M, Malik HS, Fujimori DG, Ideker T, Craik CS, Floor SN, Fraser JS, Gross JD, Sali A, Roth BL, Ruggero D, Taunton J, Kortemme T, Beltrao P, Vignuzzi M, García-Sastre A, Shokat KM, Shoichet BK, Krogan NJ (2020) A SARS-CoV-2 protein interaction map reveals targets for drug repurposing. Nature 583:459 468. https://doi.org/10.1038/s41586-020-2286-9

Hajighahramani N, Eslami M, Negahdaripour M, Ghoshoon MB, Dehshahri A, Erfani N, Heidari R, Gholami A, Nezafat N, Ghasemi Y (2019) Computational design of a chimeric epitopebased vaccine to protect against Staphylococcus aureus infections. Mol Cell Probes 46:101414. https://doi.org/10.1016/j.mcp.2019.06. 004

Hamming I, Timens W, Bulthuis MLC, Lely AT, Navis GJ, van Goor H (2004) Tissue distribution of ACE2 protein, the functional receptor for SARS coronavirus. A first step in understanding SARS pathogenesis. J Pathol 203:631-637. https://doi.org/10.1002/path.1570

Heo L, Park H, Seok C (2013) GalaxyRefine: protein structure refinement driven by side-chain repacking. Nucleic Acids Res 41(W1):W384 W388. https://doi.org/10.1093/nar/gkt458

Jackson LA, Anderson EJ, Rouphael NG, Roberts PC, Makhene M, Coler RN, McCullough MP, Chappell JD, Denison MR, Stevens LJ, Pruijssers AJ, McDermott A, Flach B, Doria-Rose NA, Corbett KS, Morabito KM, O'Dell S, Schmidt SD, Swanson PA, Padilla M, Mascola JR, Neuzil KM, Bennett H, Sun W, Peters E, Makowski M, Albert J, Cross K, Buchanan W, Pikaart-Tautges R, Ledgerwood JE, Graham BS, Beigel JH (2020) An mRNA Vaccine against SARS-CoV-2 - Preliminary Report. N Engl J Med 383: 1920-1931. https://doi.org/10.1056/nejmoa2022483

Kaur SP, Gupta V (2020) COVID-19 Vaccine: A comprehensive status report. Virus Res 288:198114. https://doi.org/10.1016/j.virusres. 2020.198114

Kim Y, Ponomarenko J, Zhu Z, Tamang D, Wang P, Greenbaum J, Lundegaard C, Sette A, Lund O, Bourne PE, Nielsen M, Peters B (2012) Immune epitope database analysis resource. Nucleic Acids Res 40:W525-W530. https://doi.org/10.1093/nar/gks438

Kim SH, Yang IY, Kim J, Lee KY, Jang YS (2015) Antimicrobial peptide LL-37 promotes antigen-specific immune responses in mice by enhancing Th17-skewed mucosal and systemic immunities. Eur J Immunol 45:1402-1413. https://doi.org/10.1002/eji.201444988

Kozakov D, Hall DR, Beglov D, Brenke R, Comeau SR, Shen Y, Li K, Zheng J, Vakili P, Paschalidis IC, Vajda S (2010) Achieving reliability and high accuracy in automated protein docking: ClusPro, PIPER, SDU, and stability analysis in CAPRI rounds 13-19. Proteins 78:3124-3130. https://doi.org/10.1002/prot.22835

Krammer F (2020) SARS-CoV-2 vaccines in development. Nature 586: 516-527. https://doi.org/10.1038/s41586-020-2798-3

Kringelum JV, Lundegaard C, Lund O, Nielsen M (2012) Reliable B cell epitope predictions: impacts of method development and improved benchmarking. PLoS Comput Biol 8:e1002829. https://doi.org/10. 1371/journal.pcbi.1002829

Lai Y, Adhikarakunnathu S, Bhardwaj K, Ranjith-Kumar CT, Wen Y, Jordan JL, Wu LH, Dragnea B, San Mateo L, Kao CC (2011b) LL37 and cationic peptides enhance TLR3 signaling by viral doublestranded RNAs. PLoS One 6:e26632. https://doi.org/10.1371/ journal.pone. 0026632
Lester SN, Li K (2014) Toll-like receptors in antiviral innate immunity. J Mol Biol 426:1246-1264. https://doi.org/10.1016/j.jmb.2013.11. 024

Livingston B, Crimi C, Newman M, Higashimoto Y, Appella E, Sidney J, Sette A (2002) A rational strategy to design multiepitope immunogens based on multiple Th lymphocyte epitopes. J Immunol 168(11):5499-5506. https://doi.org/10.4049/jimmunol.168.11. 5499

Lovell SC, Davis IW, Arendall WB, de Bakker PI, Word JM, Prisant MG, Richardson JS, Richardson DC (2003) Structure validation by $\mathrm{C} \alpha$ geometry: $\phi, \psi$ and $\mathrm{C} \beta$ deviation. Proteins $50(3): 437-450$. https://doi.org/10.1002/prot.10286

Luthy R, Bowie JU, Eisenberg D (1992) Assessment of protein models with three-dimensional profiles. Nature 356(6364):83-85. https:// doi.org/10.1038/356083a0

Mahase E (2020) Covid-19: Moderna vaccine is nearly $95 \%$ effective, trial involving high risk and elderly people shows. BMJ 371:m4471. https://doi.org/10.1136/bmj.m4471

Masters PS (2006) The Molecular biology of coronaviruses. Adv Virus Res 66:193-292. https://doi.org/10.1016/S0065-3527(06)66005-3

Michel CJ, Mayer C, Poch O, Thompson JD (2020) Characterization of accessory genes in coronavirus genomes. Virol J 17:131. https://doi. org/10.1186/s12985-020-01402-1

Mosaddeghi P, Shahabinezhad F, Dorvash M, Goodarzi M, Negahdaripour M (2020) Harnessing the non-specific immunogenic effects of available vaccines to combat COVID-19. Hum Vaccines Immunother:1-12. https://doi.org/10.1080/21645515.2020. 1833577

Moyle PM, Toth I (2013) Modern subunit vaccines: development, components, and research opportunities. ChemMedChem 8(3):360-376. https://doi.org/10.1002/cmdc.201200487

Negahdaripour M (2020) The battle against COVID-19: Where do we stand now? IJMS 45(2):81. https://doi.org/10.30476/ijms.2020. 46357

Negahdaripour M (2021) COVID-19 vaccine global access is an urgency. IJMS 46(2):79-80. https://doi.org/10.30476/ijms.2021.47336

Negahdaripour M, Eslami M, Nezafat N, Hajighahramani N, Ghoshoon MB, Shoolian E, Dehshahri A, Erfani N, Morowvat MH, Ghasemi Y (2017a) A novel HPV prophylactic peptide vaccine, designed by immunoinformatics and structural vaccinology approaches. Infect Genet Evol 54:402-416. https://doi.org/10.1016/j.meegid.2017.08. 002

Negahdaripour M, Golkar N, Hajighahramani N, Kianpour S, Nezafat N, Ghasemi Y (2017b) Harnessing self-assembled peptide nanoparticles in epitope vaccine design. Biotechnol Adv 35(5):575-596. https://doi.org/10.1016/j.biotechadv.2017.05.002

Negahdaripour M, Nezafat N, Eslami M, Ghoshoon MB, Shoolian E, Najafipour S, Morowvat MH, Dehshahri A, Erfani N, Ghasemi Y (2018) Structural vaccinology considerations for in silico designing of a multi-epitope vaccine. Infect Genet Evol 58:96-109. https://doi. org/10.1016/j.meegid.2017.12.008

Negahdaripour M, Nezafat N, Heidari R, Erfani N, Hajighahramani N, Ghoshoon MB, Shoolian E, Rahbar MR, Najafipour S, Dehshahri A (2020) Production and preliminary in vivo evaluations of a novel in silico-designed L2-based potential HPV vaccine. Curr Pharm Biotechnol 21:316-324. https://doi.org/10.2174/ 1389201020666191114104850

O'Hagan DT, De Gregorio E (2009) The path to a successful vaccine adjuvant - 'The long and winding road'. Drug Discov Today 14: 541-551. https://doi.org/10.1016/j.drudis.2009.02.009

O'Neill LA, Bowie AG (2007) The family of five: TIR-domaincontaining adaptors in Toll-like receptor signalling. Nat Rev Immunol 7:353-364. https://doi.org/10.1038/nri2079

Owji H, Negahdaripour M, Hajighahramani N (2020) Immunotherapeutic approaches to curtail COVID-19. Int 
Immunopharmacol 88:106924. https://doi.org/10.1016/j.intimp. 2020.106924

Qiu M, Shi Y, Guo Z, Chen Z, He R, Chen R, Zhou D, Dai E, Wang X, Si B (2005) Antibody responses to individual proteins of SARS coronavirus and their neutralization activities. Microb Infect 7:882-889. https://doi.org/10.1016/j.micinf.2005.02.006

Ramasamy MN, Minassian AM, Ewer KJ, Flaxman AL, Folegatti PM, Owens DR, Voysey M, Aley PK, Angus B, Babbage G (2021) Safety and immunogenicity of ChAdOx $1 \mathrm{nCoV}-19$ vaccine administered in a prime-boost regimen in young and old adults (COV002): a single-blind, randomised, controlled, phase 2/3 trial. Lancet 396: 1979-1993. https://doi.org/10.1016/S0140-6736(20)32466-1

Reed SG, Hsu F-C, Carter D, Orr MT (2016) The science of vaccine adjuvants: advances in TLR4 ligand adjuvants. Curr Opin Immunol 41:85-90. https://doi.org/10.1016/j.coi.2016.06.007

Ren Y, Shu T, Wu D, Mu J, Wang C, Huang M, Han Y, Zhang XY, Zhou W, Qiu Y, Zhou X (2020) The ORF3a protein of SARS-CoV-2 induces apoptosis in cells. Cell Mol Immunol 17:881-883. https:// doi.org/10.1038/s41423-020-0485-9

Saha S, Raghava GPS (2006) AlgPred: prediction of allergenic proteins and mapping of IgE epitopes. Nucleic Acids Res 34:W202-W209. https://doi.org/10.1093/nar/gkl343

Samad A, Ahammad F, Nain Z, Alam R, Imon RR, Hasan M, Rahman MS (2020) Designing a multi-epitope vaccine against SARS-CoV2: an immunoinformatics approach. J Biomol Struct Dyn 1-17. https://doi.org/10.1080/07391102.2020.1792347

Samuel CE (2001) Antiviral actions of interferons. Clin Microbiol Rev 14:778-809

Shin WH, Lee GR, Heo L, Lee H, Seok C (2014) Prediction of protein structure and interaction by GALAXY protein modeling programs. Bio Design 2:1-11

Siu KL, Yuen KS, Castaño-Rodriguez C, Ye ZW, Yeung ML, Fung SY, Yuan S, Chan C-P, Yuen KY, Enjuanes L (2019) Severe acute respiratory syndrome coronavirus ORF3a protein activates the NLRP3 inflammasome by promoting TRAF3-dependent ubiquitination of ASC. FASEB J 33:8865-8877. https://doi.org/ 10.1096/fj.201802418R

Soria-Guerra RE, Nieto-Gomez R, Govea-Alonso DO, Rosales-Mendoza $S$ (2015) An overview of bioinformatics tools for epitope prediction: implications on vaccine development. J Biomed Inform 53:405414. https://doi.org/10.1016/j.jbi.2014.11.003

Suresh R, Mosser DM (2013) Pattern recognition receptors in innate immunity, host defense, and immunopathology. Adv Physiol Educ 37:284-291. https://doi.org/10.1152/advan.00058.2013

Vakili B, Eslami M, Hatam GR, Zare B, Erfani N, Nezafat N, Ghasemi Y (2018) Immunoinformatics-aided design of a potential multi-epitope peptide vaccine against Leishmania infantum. Int J Biol Macromol 120:1127-1139. https://doi.org/10.1016/j.ijbiomac

van der Burg SH, Bijker MS, Welters MJ, Offringa R, Melief CJ (2006) Improved peptide vaccine strategies, creating synthetic artificial infections to maximize immune efficacy. Adv Drug Deliv Rev 58: 916-930. https://doi.org/10.1016/j.addr.2005.11.003

Walsh EE, Frenck RW, Falsey AR, Kitchin N, Absalon J, Gurtman A, Lockhart S, Neuzil K, Mulligan MJ, Bailey R, Swanson KA, Li P, Koury K, Kalina W, Cooper D, Fontes-Garfias C, Shi P-Y, Türeci Ö, Tompkins KR, Lyke KE, Raabe V, Dormitzer PR, Jansen KU, Şahin U, Gruber WC (2020) Safety and immunogenicity of two RNA-based Covid-19 vaccine candidates. N Engl J Med. https:// doi.org/10.1056/NEJMoa2027906

Wang P, Sidney J, Dow C, Mothé B, Sette A, Peters B (2008) A systematic assessment of MHC class II peptide binding predictions and evaluation of a consensus approach. PLoS Comput Biol 4(4): e1000048-e1000048. https://doi.org/10.1371/journal.pcbi.1000048

WHO website. https://covid19.who.int/. Accessed 22 May 2021

Wiederstein M, Sippl MJ (2007) ProSA-web: interactive web service for the recognition of errors in three-dimensional structures of proteins. Nucleic Acids Res 35:W407-W410. https://doi.org/10.1093/nar/ gkm290

Yang J, Zhang Y (2015) I-TASSER server: new development for protein structure and function predictions. Nucleic Acids Res 43:W174 W181. https://doi.org/10.1093/nar/gkv342

Yang J, Yan R, Roy A, Xu D, Poisson J, Zhang Y (2015) The I-TASSER Suite: protein structure and function prediction. Nat Methods 12:78. https://doi.org/10.1038/nmeth.3213

Yang Z, Bogdan P, Nazarian S (2021) An in-silico deep learning approach to multi-epitope vaccine design: A SARS-CoV-2 case study. Sci Rep 11. https://doi.org/10.21203/rs.3.rs-36528/v1

Zeng W, Liu G, Ma H, Zhao D, Yang Y, Liu M, Mohammed A, Zhao C, Yang Y, Xie J (2020) Biochemical characterization of SARS-CoV-2 nucleocapsid protein. Biochem Biophys Res Commun 527:618 623. https://doi.org/10.1016/j.bbrc.2020.04.136

Zhou P, Yang XL, Wang XG, Hu B, Zhang L, Zhang W, Si HR, Zhu Y, Li B, Huang CL, Chen HD, Chen J, Luo Y, Guo H, Jiang RD, Liu MQ, Chen Y, Shen XR, Wang X, Zheng XS, Zhao K, Chen QJ, Deng F, Liu LL, Yan B, Zhan FX, Wang YY, Xiao GF, Shi ZL (2020) A pneumonia outbreak associated with a new coronavirus of probable bat origin. Nature 579:270-273. https://doi.org/10.1038/ s41586-020-2012-7

Publisher's Note Springer Nature remains neutral with regard to jurisdictional claims in published maps and institutional affiliations. 